\title{
Uncertainty in Environmental Risk Assessment: Implications for Risk-Based Management of River Basins
}

\author{
Ad MJ Ragas, * Mark AJ Huijbregts, Irmgard Henning-de Jong, and Rob SEW Leuven \\ Department of Environmental Science, Institute for Water and Wetland Research, Faculty of Science, Radboud University, PO Box 9010, \\ 6500 GL Nijmegen, The Netherlands
}

(Received 14 September 2008; Accepted 2 October 2008)

\section{EDITOR'S NOTE:}

This is 1 of 12 papers prepared by participants attending the workshop "Risk Assessment in European River Basins-State of the Art and Future Challenges" held in Liepzig, Germany on 12-14 November 2007. The meeting was organized within the framework of the European Commission's Coordination Action RISKBASE program. The objective of RISKBASE is to review and synthesize the outcome of European Commission FP4-FP6 projects, and other major initiatives, related to integrated risk assessment-based management of the water/ sediment/soil environment at the river basin scale.

\section{ABSTRACT}

Environmental risk assessment is typically uncertain due to different perceptions of the risk problem and limited knowledge about the physical, chemical, and biological processes underlying the risk. The present paper provides a systematic overview of the implications of different types of uncertainty for risk management, with a focus on risk-based management of river basins. Three different types of uncertainty are distinguished: 1) problem definition uncertainty, 2) true uncertainty, and 3) variability. Methods to quantify and describe these types of uncertainty are discussed and illustrated in 4 case studies. The case studies demonstrate that explicit regulation of uncertainty can improve risk management (e.g., by identification of the most effective risk reduction measures, optimization of the use of resources, and improvement of the decision-making process). It is concluded that the involvement of nongovernmental actors as prescribed by the European Union Water Framework Directive (WFD) provides challenging opportunities to address problem definition uncertainty and those forms of true uncertainty that are difficult to quantify. However, the WFD guidelines for derivation and application of environmental quality standards could be improved by the introduction of a probabilistic approach to deal with true uncertainty and a better scientific basis for regulation of variability.

Keywords: Uncertainty Variability Risk assessment River management Environmental quality standards

\section{INTRODUCTION}

Environmental risk assessment is an organized process that aims to estimate the likelihood of adverse health outcomes after exposure to environmental stressors (USPCCRARM 1997). The outcome of the assessment forms the basis for a management decision; for example, whether or not to take action and reduce the risk. Risk assessment is a knowledgebased process (i.e., scientific knowledge about the use, emission, fate, exposure, and/or effects of the environmental stressors is gathered and reviewed to produce a risk estimate). The outcome of the assessment is a qualitative or quantitative description of the risk that is typically uncertain due to different perceptions of the risk problem and limited knowledge about the physical, chemical, and biological processes underlying the risk.

Uncertainty in the risk assessment process may have a profound impact on the management decision. This can best be illustrated with an example. When stakes are high (e.g., in the safety management of a nuclear power plant), the probability at which an accident will be considered "acceptable by society" will be much smaller than when stakes are relatively low (e.g., for traffic accidents). It should be noted that, besides uncertainty, there are many other factors that

* To whom correspondence may be addressed: A.Ragas@science.ru.nl Published on the Web 10/9/2008. may influence the acceptability of risks (e.g., voluntary vs imposed risks) (Slovic 2001).

Risk assessment is an important aspect of river basin management as laid down in the European Union Water Framework Directive (WFD; Directive 2000/60/EC). Examples are the assessment of point source discharges on water systems and the evaluation of the chemical status of a water body. Environmental quality standards (EQSs), such as water and sediment standards, play an important role in this risk assessment process. They are generally compared with predicted or measured concentrations to obtain an indication of the risk. If the EQS is uncertain, the risk indicator will also be uncertain. This also holds for the predicted or measured concentration.

Awareness is growing that uncertainty may have important implications for risk management (e.g., in risk-based management of river basins). However, a systematic overview of the implications of uncertainty for risk-based management of river basins is currently lacking. The present paper aims to provide such an overview. It does so by summarizing 4 case studies on uncertainty that were performed by our research group. The paper starts with a typology of the different types of uncertainty and how these can be quantified or described. Then the case studies are presented. The paper concludes with a discussion section in which the implications of the different types of uncertainty for risk management are outlined. 


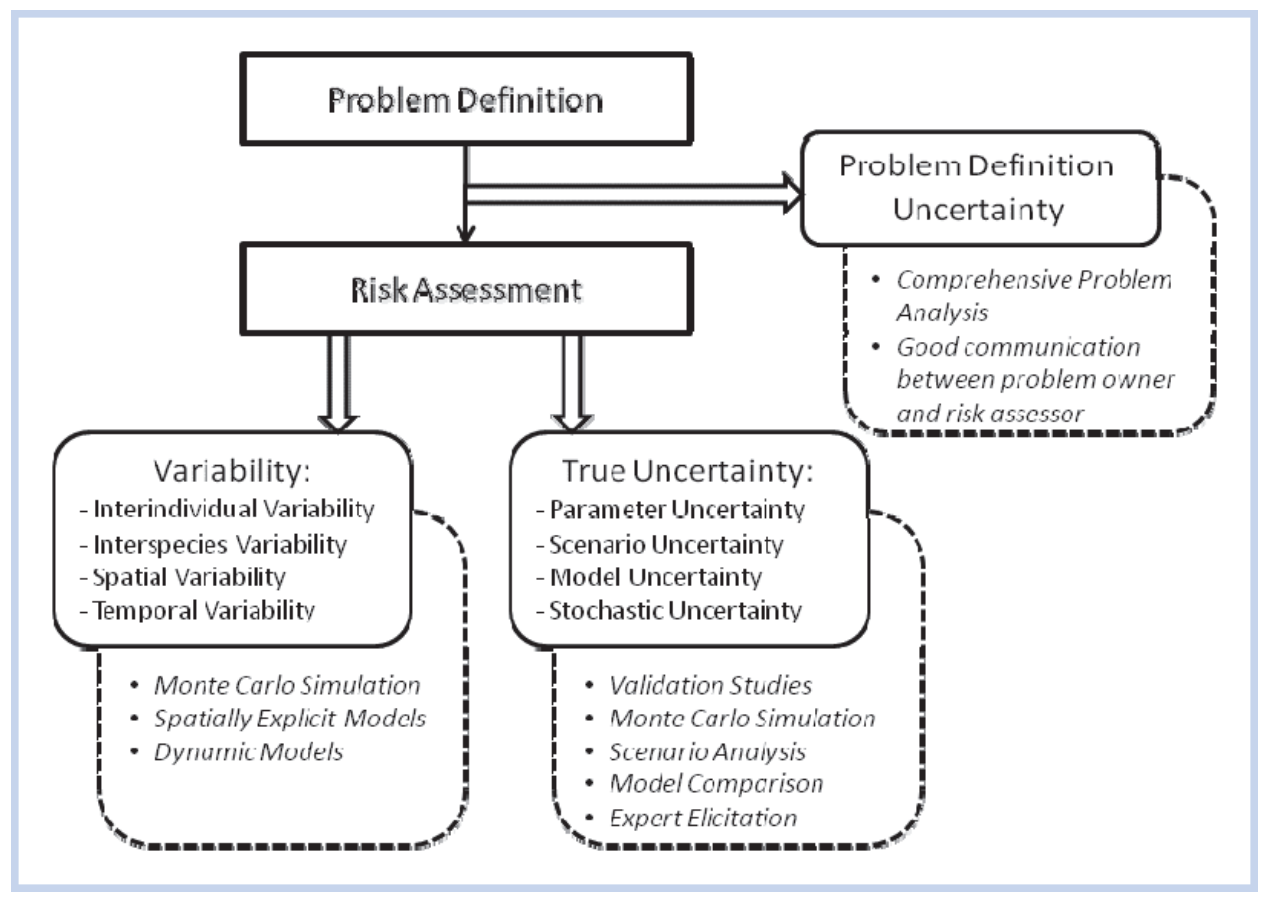

Figure 1. Overview of different types of uncertainty (solid boxes with rounded corners) in the risk assessment process (square boxes), and methods that can be applied to describe or quantify them (dashed boxes with rounded corners).

\section{UNCERTAINTY}

The term uncertainty is generally used as an overarching term to indicate that the results of a risk assessment are not necessarily true or applicable to a particular situation. Several authors have pointed out that there are many different types of uncertainty (Funtowicz and Ravetz 1990; Morgan and Henrion 1990; Renn 1998). It is important to distinguish between these types of uncertainty because they may have different interpretations and implications for risk management. Here, we distinguish 1) problem definition uncertainty, 2) true uncertainty, and 3) variability. Figure 1 illustrates the different types of uncertainty and some methods used to describe or quantify them.

\section{Problem definition uncertainty}

Problem definition uncertainty may arise when the definition of the management problem differs from that of the scientific risk assessment problem. For example, the management problem may consist of the protection of aquatic ecosystems. This problem can be translated into several scientific problems, one being the determination of safe concentration levels for individual chemicals. Once these safe concentration levels have been determined and realized, the management problem has been partially solved. However, even if the safe concentration levels for individual chemicals are met, the aquatic ecosystem may still be in a poor condition, for example, because it is affected by multiple chemicals (mixture toxicity) or other stressors, such as low water levels, that were not considered in the scientific analysis.

\section{True uncertainty}

Uncertainty due to a lack of knowledge is referred to as true uncertainty in the present paper. The level of knowledge can vary from complete ignorance to imprecision. Examples of true uncertainty are (model) assumptions, simplifications, approximate equations, and imprecise measurements. An important characteristic of true uncertainty is that it can be reduced by additional research. Thus, an analysis of true uncertainty does not only provide information about the extent of the uncertainty, but also in options to reduce it. In other words, the results of an uncertainty analysis can be used to plan new research.

Many different techniques exist to quantify true uncertainty. The best option is validation research (i.e., comparison of the risk assessment outcome with field measurements). However, this is often unfeasible or only partially feasible. Other options include propagation of parameter uncertainty (e.g., by means of Monte Carlo simulation), model comparison, scenario analysis, and expert elicitation. Which technique to use depends on where in the risk assessment the major source(s) of uncertainty are located. This can best be illustrated with an example. An input-output uncertainty analysis, such as a Monte Carlo simulation, may show that parameter uncertainty is relatively small, but this result becomes meaningless if the assumptions on which the model is based are highly uncertain. A good uncertainty analysis should therefore thoroughly investigate the sources of uncertainty on all levels of the assessment: Model assumptions and equations, the input scenario(s), and uncertainty in the input parameters (Van der Sluijs et al. 2005). An inputoutput analysis of uncertainty suffices only if it can be shown that the other sources of uncertainty do not dominate.

Monte Carlo simulation and similar numerical or analytical techniques are well established and widely used to propagate true uncertainty in model parameters into the output (Hammersley and Handscomb 1964; NRC 1975; McKone and Ryan 1989). The popularity of these techniques can be explained by the fact that they produce a quantitative estimate of the amount of uncertainty (i.e., a probability distribution). However, it should be noted that the probability distribution only provides a realistic picture of the true uncertainty if 1) the probability distribution of the input 
parameters are appropriately defined (i.e., the shape and parameters), 2) correlations between input parameters are appropriately accounted for, and 3) other sources of true uncertainty do not dominate. Techniques to estimate scenario uncertainty are also fairly well established and regularly used, such as by compiling different (input) scenarios and comparison of the results (Huijbregts et al. 2003; Webster et al. 2003; Van Asselt et al. 2005). However, the allocation of probabilities to input scenarios can be problematic, which may impede the expression of the uncertainty in terms of probabilities. Scenario analysis is often used to obtain an impression of the range of the uncertainty, but it is less suitable to obtain a full quantitative description. Techniques to estimate uncertainty in model assumptions and equations include model comparisons and expert elicitation. Model comparisons can provide useful insights on model differences and reveal sources of uncertainty (e.g., Huijbregts et al. 2005), but part of the uncertainty may remain unnoticed if the models included are based on similar assumptions (Ragas et al. 1999). Furthermore, close agreement of model predictions does not prove that the models are correct; it may also be explained by pure chance or by the fact that the models were calibrated to similar data sets (Thiessen et al. 1997). Finally, expert elicitation may be used to obtain an impression of the true uncertainty, but there are many methodological issues, such as selection of the experts, framing of the questions, and reproducibility, that can make it difficult to interpret and quantify the results. For example, Evans et al. (1994) showed that the uncertainty in the estimated cancer risk due to benzene exposure is very sensitive to extreme opinions of one or more experts.

It follows from the above that quantification of true uncertainty in risk assessment is not always feasible or only partially feasible. Within this context, authors have distinguished several levels of true uncertainty. Vercelli (1995) introduced the terms hard and soft uncertainty. The latter term refers to uncertainty that can be expressed in a probability distribution whereas the former is reserved for qualitative descriptions of uncertainty. Ragas et al. (1999) introduced the terms operational and fundamental uncertainty to refer to uncertainty resulting from input parameters, and model structure and assumptions, respectively. Whichever system is used to characterize true uncertainty, they all recognize that uncertainty cannot always be expressed in a simple statistical probability distribution. This implies that a thorough uncertainty analysis should include an assessment of the nonquantifiable sources of uncertainty and that risk management must find ways to deal with these nonquantifiable sources.

\section{Variability}

Variability is a phenomenon of the real world; for example, the variation of pollutant concentrations in space or in time. Another example is the variation in sensitivity or exposure between the individuals of a population or the species of an ecosystem. These types of variability are generally referred to as spatial, temporal, interindividual, and interspecies variability, respectively. Variability can be described by means of scientific research, but it cannot be reduced by it. Variability becomes an issue in risk assessment when aggregate measures are used to describe reality; for example, population indexes to characterize a group of individuals or a spatial average to characterize the concentration of a chemical in an area (Leuven and Poudevigne 2002). For example, when the spatial average is known but the underlying, disaggregate data are missing or lost in the aggregation process, the question may arise of how much the concentration in a specific location will deviate from the spatial average (Hollander 2008). Variability analysis can answer questions such as "Where and how much is the EQS in an area exceeded?" or "How many and which individuals exceed the Acceptable Daily Intake?" This information is useful to check compliance with regulations, but even more so to identify areas at risk or individuals at risk, so appropriate management actions can be taken.

True uncertainty and variability are often considered simultaneously in risk assessment studies. The 2 main reasons for this are 1) uncertainty and variability are traditionally quantified together in a probabilistic risk assessment (i.e., both can be expressed using a variance), and 2) variability can result in uncertainty, depending on the perspective taken. Notwithstanding the fact that true uncertainty and variability have many characteristics in common, they are 2 distinct phenomena that have different interpretations and implications for risk managers (Bogen and Spear 1987; Frey 1992; NRC 1994; Kelly and Campbell 2000). An analysis of true uncertainty provides insight into the likelihood of an adverse event based on the available knowledge, whereas an analysis of variability provides insight into the magnitude of an event, such as the number of individuals exceeding a threshold (interindividual variability), the spatial area exceeding a concentration limit (spatial variability), or the amount of time during which an EQS is violated (temporal variability).

\section{CASE STUDIES}

\section{Case I: True uncertainty and interindividual variability in EQSs}

Environmental quality standards indicate a concentration level at which adverse effects on human health and ecosystems are expected to be absent or highly unlikely. Ragas (2000) quantified the level of true uncertainty and interindividual variability in Dutch EQSs for water, air, and soil. It should be stressed that the models and assumptions used in this study were not fully in line with the new guidelines of the WFD, but comparable procedures were used. It is therefore likely that the estimates give a good indication of the level of true uncertainty and interindividual variability in EQSs that are derived according to the WFD guidelines (Lepper 2005). Relevant differences are discussed in the last paragraph of this case study.

The methods used to quantify true uncertainty and interindividual variability consisted of 1) Monte Carlo simulation, 2) model comparisons, and 3) expert judgment. The results of the analysis were expressed in uncertainty factors (UFs) that were calculated as the ratio between the 95th and 50th percentile of the predicted output distributions. Separate uncertainty factors were estimated for

1. EQSs derived to protect humans against genotoxic carcinogens;

2. EQSs derived to protect humans against substances with a threshold effect;

3. EQSs to protect ecosystems.

The uncertainty factors were derived by quantification of uncertainty and variability in different steps of the derivation process of EQSs (e.g., exposure and effect modeling). 
Table 1. Uncertainty factors for the derivation of Dutch environmental quality standards (Ragas 2000)

\begin{tabular}{|c|c|c|c|c|c|}
\hline & \multicolumn{2}{|c|}{ Exposure Modeling } & \multicolumn{2}{|c|}{ Effect Modeling } & \multirow{2}{*}{$\begin{array}{l}\text { Total } \\
\text { TUF }^{\mathrm{e}}\end{array}$} \\
\hline & OUF $^{a}$ & FUF $^{b, c}$ & OUF $^{b}$ & FUF $^{b, d}$ & \\
\hline \multicolumn{6}{|c|}{ Human health standards } \\
\hline \multicolumn{6}{|c|}{ Genotoxic carcinogens } \\
\hline $\mathrm{GM}^{\mathrm{f}}$ & 1.6 & 2.0 & 59 & $1.4 \times 10^{10}$ & $1.4 \times 10^{10}(64)$ \\
\hline $\operatorname{MAX}^{g}$ & 1.7 & 5.0 & 96 & $780 \times 10^{10}$ & $820 \times 10^{10}(130)$ \\
\hline \multicolumn{6}{|c|}{ Substances with threshold } \\
\hline $\mathrm{GM}^{\mathrm{f}}$ & 1.6 & 2.0 & & 8.4 & 9.8 \\
\hline MAX ${ }^{g}$ & 1.7 & 5.0 & & 19 & 30 \\
\hline \multicolumn{6}{|c|}{ Ecological standards } \\
\hline $\mathrm{GM}^{f}$ & - & - & - & - & 6.5 \\
\hline MAX & - & - & - & - & 33 \\
\hline
\end{tabular}

${ }^{\text {a }}$ OUF = operational uncertainty factor; operational uncertainty covers true uncertainty and interindividual variability in input parameters and was quantified by Monte Carlo simulation.

${ }^{b}$ FUF = fundamental uncertainty factor; fundamental uncertainty covers true uncertainty in model structure, equations, and assumptions.

' Quantified by expert judgment.

d Quantified by comparison of different dose-response models.

e TUF = total uncertainty factor (see text for calculation; values in parentheses indicate the total uncertainty if only the conventional linear 2stage dose-response model is considered).

${ }^{f} \mathrm{GM}=$ geometric mean.

${ }^{\mathrm{g}} \mathrm{MAX}=$ maximum.

For human exposure modeling and effect modeling of genotoxic carcinogens, operational uncertainty (input uncertainty and variability quantified by means of Monte Carlo simulation), and fundamental uncertainty (model uncertainty quantified by means of model comparisons or expert judgment) were quantified separately. For effect modeling of substances with a threshold and ecological effect modeling, an overall uncertainty factor was estimated that covered operational as well as fundamental uncertainty. Uncertainty factors for different assessment steps and different types of uncertainty (operational and fundamental) were combined under the assumption that uncertainty and variability are propagated through multiplicative relationships and can be characterized by independent log-normal distributions (Slob 1994).

The results of the analysis are presented in Table 1. For each EQS type, a geometric mean and a maximum UF are specified. This variation in UFs is caused by the fact that the level of estimated true uncertainty and interindividual variability varies with the substances assessed, the models applied, and the experts selected. The difference between the geometric mean and maximum UF gives an indication of the range in the uncertainty assessment (the level of uncertainty in the uncertainty assessment itself).

The UFs in Table 1 give an indication of the range in possible outcomes relative to the most likely value (the geometric mean of the output distribution). This provides information on the level of true uncertainty and interindividual variability in a probabilistic assessment. It allows statements such as "There is a probability of $5 \%$ that the true human NOEC of a randomly selected individual is a factor 8.4 higher than the estimated median NOEC" (i.e., for effect assessment of substances with a threshold).
Table 1 clearly shows that effect modeling of genotoxic carcinogens is associated with the highest UFs. Detailed analysis of the results showed that these high UFs are mainly caused by model uncertainty (i.e., the choice of an appropriate dose-response model) and high to low dose extrapolation (Ragas 2000). Both are sources of true uncertainty. These results indicate that the largest reduction in uncertainty in probabilistic EQSs for genotoxic carcinogens can be obtained by unraveling the nature of the dose-response relationship in the low-dose region.

The UFs of the remaining 2 EQS types are of comparable magnitude (i.e., a geometric mean uncertainty factor of 6.5 for ecosystem protection and of 9.8 for human protection against substances with a threshold). Noticeable are the relatively low UFs for human exposure assessment compared to human effect assessment. This indicates that exposure processes are generally better understood and less variable than effect processes. In a similar way, Ragas (2000) showed that substance dispersion and mixing processes in water are generally much better understood than effect processes.

The values in Table 1 provide insight in the range of possible outcomes in a probabilistic assessment, but they do not tell how this probabilistic prediction compares with the deterministic EQS. This latter information is more relevant for risk managers because it indicates the level of conservativeness of the EQS. Ragas (2000) performed this comparison for the virtually safe dose (the dose that corresponds to a $1 \times 10^{-6}$ lifetime cancer risk) of 3 genotoxic carcinogens. Using the default linear 2-stage dose-response model, the probability that the deterministic virtually safe dose exceeds the probabilistic virtually safe dose was $7.0 \%, 46 \%$, and $11.6 \%$ for acrylamide, chlordane, and DDT, respectively. These results show that although the current procedures are relatively conservative, there is a realistic probability of 


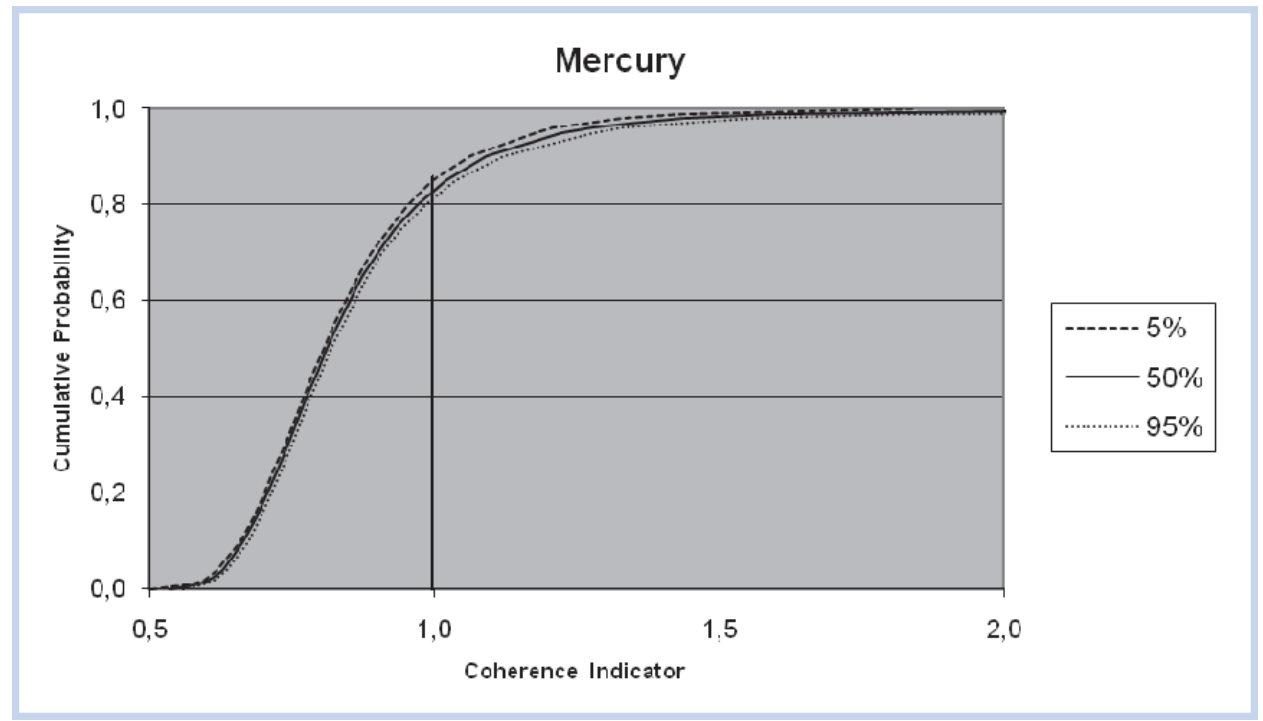

Figure 2. Parameter uncertainty and interindividual variability in the coherence indicator of mercury.

underestimating the true risk. Furthermore, it is remarkable that the level of conservativeness varies considerably between different substances.

It should be noted that the level of conservativeness in water and sediment standards derived according to the WFD (Lepper 2005) is likely to be higher than that in the virtually safe doses for genotoxic carcinogens. Two reasons are behind this:

1. WFD standards are based on multiple protection targets, such as the pelagic community, the benthic community, secondary poisoning of predators, adverse human health effects after consumption of fish, and drinking water abstraction (Lepper 2005). The most critical protection target determines the level of the WFD standard, which implies that the WFD standard may be very conservative from the perspective of noncritical protection targets.

2. Ecological WFD standards are more or less derived according to the procedure applied in the uncertainty analysis (i.e., the application of species sensitivity distributions [SSDs]). However, WFD standards for the protection of human health, such as standards for fish consumption and drinking water abstraction, are derived using relatively conservative assumptions: 1) for fish consumption as well as drinking water consumption, the maximum intake of a substance is set at $10 \%$ of the human threshold level, 2) humans consume $115 \mathrm{~g}$ of fish per day, 3) conservative bioaccumulation and biomagnification factors are used to translate the concentration in fish to a corresponding water concentration, and 4) humans consume $2 \mathrm{~L}$ of water per day. These assumptions increase the level of conservativeness of the WFD standards for human health protection.

\section{Case II: True uncertainty and interindividual variability in human exposure modeling}

The impact of true uncertainty and variability in model parameters is often quantified by means of Monte Carlo simulation. In conventional Monte Carlo simulation, true uncertainty and variability are mixed in one estimate of variance; for example, the operational UFs listed in Table 1. This mixing impedes an unambiguous interpretation of the results. If true uncertainty dominates, the output indicates the likelihood that the entire population is at risk. If interindividual variability dominates, the output indicates the variation in the population and can be used to quantify the population fraction exceeding a certain threshold. It is therefore important to know which part of the output variation reflects true uncertainty and which part reflects interindividual variability.

Ragas et al. (2008) performed a case study on human exposure modeling in which they separated the impact of true uncertainty and interindividual variability by means of nested Monte Carlo simulation. Model parameters were classified as uncertain, variable, or both. The model that was used (NORMTOX; Ragas and Huijbregts 1999; Ragas et al. 2008), predicts lifetime-averaged daily oral and inhalatory intake of environmental contaminants through food, soil, air, and drinking and swimming water. The model was developed to test the coherence of Dutch EQSs. A set of EQSs is called coherent if simultaneous exposure to different environmental media that are all polluted up to the applicable EQS does not result in exceeding the acceptable or tolerable daily intake. To this end, a coherence indicator was used which equals the ratio between the intake distribution predicted by NORMTOX and the applicable acceptable daily intake or tolerable daily intake. If the coherence indicator exceeds unity, the EQSs are incoherent. The aim of the case study was to separate the impact of parameter uncertainty and interindividual variability in coherence predictions of Dutch EQSs.

Figure 2 gives the results for mercury. The plot shows 3 lines, clustering close together. Each line can be considered to represent one hypothetical population. The variance within one line, reflected by the slope, represents interindividual variability in exposure within the Dutch population. The variance between the lines, in other words between possible populations, represents the impact of parameter uncertainty. In Figure 2, only the 5th, 50th, and 95th percentile populations out of 100 simulated populations are given.

From the fact that the lines in Figure 2 cluster close together, it can be deduced that parameter uncertainty is relatively small. The overall variance in the predicted 
Table 2. Coefficients of variation quantifying the impact of interindividual variability and parameter uncertainty in exposure predictions for mercury per exposure pathway

\begin{tabular}{|l|c|c|c|}
\hline Route & Var $^{\mathbf{a}}$ & Unc $^{\mathbf{b}}$ & Var/Unc \\
\hline Total & 0.24 & 0.01 & 28 \\
\hline Soil & 0.42 & 0.05 & 8.4 \\
\hline Swimming water & 1.11 & 1.04 & 1.1 \\
\hline Food and drinking water & 0.37 & 0.01 & 25 \\
\hline
\end{tabular}

a Variability; defined as the average coefficients of variation of the 100 simulated populations.

${ }^{b}$ Uncertainty at the mean value of the 100 simulated populations, expressed by its coefficients of variation.

exposure, and therefore in the coherence indicator, is dominated by interindividual variability. This can be explained by the fact that food is the major exposure route for mercury, in combination with the fact that food intake parameters have relatively large interindividual variability and little uncertainty. Uncertainty in food intake parameters is small because interindividual variability was estimated based on fitting a frequency distribution over a large number of individual intake data. The high number of participants in the food survey (6250 individuals) explains the relatively small uncertainty.

More detailed analysis of the case study results showed that the ratio between parameter uncertainty and interindividual variability differs between the exposure routes (Table 2). Interindividual variability clearly dominates exposure through food and, to a lesser extent, soil. However, uncertainty dominates for exposure through swimming water. This implies that additional research on oral intake of swimming water may significantly improve predictions for those substances for which this intake route is important. It illustrates how the ratio between uncertainty and interindividual variability can serve as guidance for decision makers. When uncertainty is the dominant source of variance in the output, one can decide to investigate parameters for which the model is most sensitive in more detail. If interindividual variability is the major source of variance it will be useful to search for parameter values that explain high exposure and can be used to identify subpopulations at risk. This information can subsequently be used to take specific actions for exposure reduction.

The case study emphasizes the relevance of distinguishing between parameter uncertainty and interindividual variability in risk assessment. This distinction is also relevant for risk assessment in river basins. An example is a risk assessment for the consumption of contaminated fish. The intake of fish will vary between individuals, while other exposure parameters (e.g., the contaminant concentration, degradation, or absorption) may be uncertain. The distinction is not only relevant for exposure assessment but also for effect assessment. An analysis of the interindividual variability in sensitivity may support the identification of sensitive subpopulations (Dorne et al. 2005, 2007). Finally, the distinction between uncertainty and variability is also relevant in ecological risk assessment. Here, it is generally not the variability between individuals that is of interest, but the variability between species. Separation of parameter uncertainty and interspecies variability is already common practice in the application of
SSDs, where the fraction of species at risk and the likelihood of this risk can be quantified separately (Aldenberg and Jaworska 2000).

\section{Case III: Spatial and temporal variability in environmental quality standards}

The previous case study emphasized the importance of distinguishing between true uncertainty and variability. This case study focused on one particular type of variability (i.e., interindividual variability). Interpretation of interindividual variability is relatively straightforward because it relates directly to the protection target; an analysis of interindividual variability indicates the number of individuals or the population fraction at risk of exceeding a certain threshold value. Interspecies variability in ecological risk assessment also relates fairly directly to the protection target, indicating the number or fraction of species at risk.

Interpretation of variability becomes more complicated when it originates from spatial or temporal variability. Ragas (1999) illustrated this in a case study where he applied 6 different discharge mixing models to 4 different real life discharge situations. The case study not only included spatial and temporal variability, but also model uncertainty and variability in national regulations (e.g., EQSs).

For each model and discharge situation, maximum allowable annual pollutant loads were calculated according to the 1998 water regulations applicable in Germany, the United Kingdom, the Netherlands, and the United States of America. The results revealed differences in pollutant loads due to model selection and national regulations that for some discharge situations exceeded a factor of 25 (Figure 3). These differences are caused by several factors, such as 1) model differences, 2) differences in EQSs between states, 3) differences in mixing zone regulations, and 4) differences in temporal specification of EQSs (e.g., whether the EQS should be maintained during $50 \%, 90 \%$, or $100 \%$ of the time).

The fact that part of the variation was caused by different ways of dealing with spatial and temporal characteristics of EQSs, raises the question of how EQSs should be specified in terms of space and time. In other words, how should spatial and temporal variability in concentrations be dealt with when maintaining EQSs? The answer to this question is less obvious than for interindividual and interspecies variability because the relationship between temporal and spatial variability on the one hand and the protection target on the other (i.e., human health or ecosystems) is not always clear. It raises questions such as "Is the aquatic ecosystem affected in an unacceptable way if the EQS is exceeded during $10 \%$ of the time or over $25 \%$ of the river width?" Similar questions can be raised in relation to human health protection, such as "Do people suffer adverse health effects if the acceptable daily intake is exceeded during 25\% of the time?" Regarding temporal variability, one could argue that it is unnecessary to maintain an EQS that is based on long-term impacts, such as bioaccumulation or cancer, over $90 \%$ of the time. In this case, it seems more plausible to check compliance of an EQS against the long-term average, such as the annual average. However, for substances that act relatively quickly, such as most pesticides, a violation of the EQS during $10 \%$ of the time (e.g., 36 days of the year) may result in unacceptable effects. Regarding spatial variability, the main question is at what size of the mixing zone the aquatic ecosystem and human health may be affected. It is remarkable how little 


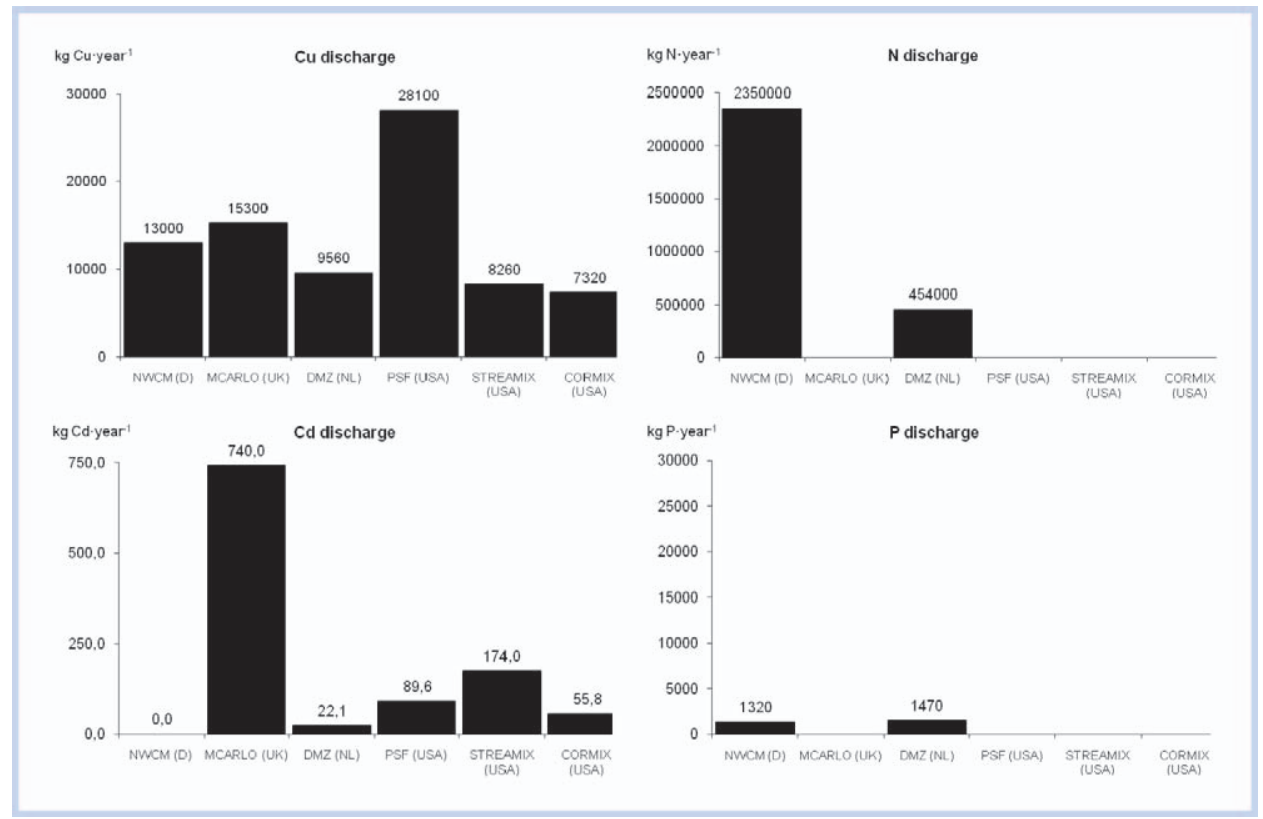

Figure 3. Maximum allowable pollutant loads (in $\mathrm{kg} / \mathrm{y}$ ) for a copper (Cu), nitrogen (N), cadmium (Cd), and phosphate (P) discharge calculated with 6 different discharge mixing models: NWCM, MCARLO, DMZ, PSF, STREAMIX, and CORMIX. Each model was applied in combination with the national EQSs and mixing zone regulations: German regulations for NWCM (D), English for MCARLO (UK), Dutch for DMZ (NL), and American for PSF, STREAMIX, and CORMIX (USA). Missing values are caused by a lack of national regulations (e.g., if no EQS is available).

empirical data is available on the impact of mixing zones on aquatic ecosystems and human health, because (eco)toxicological tests generally apply homogeneous exposure regimes. Most likely, the impact of a mixing zone will differ between species. For example, the relatively high concentrations in the mixing zone may have relatively little impact on passing organisms like migrating fish, but sessile and slowly moving organisms like water plants and mussels may be seriously affected.

The draft European Union Directive of on environmental quality standards (CEC 2006) specifies an annual arithmetic mean concentration and maximum acceptable concentration EQS. These values refer to 2 different temporal dimensions: An annual average and a maximum concentration that should not be exceeded at any time. The latter standard poses a problem from a statistical perspective: If more samples are taken, the probability that one of the samples exceeds the maximum acceptable concentration EQS increases. The water manager is punished for gathering extra information. It would be more appropriate if the maximum acceptable concentration EQS would be specified as a percentile value of a time series (e.g., the 97.5th percentile). In this case, the water manager at least has the chance to prove that a violation was an incident (i.e., by gathering additional data). Maintenance of the maximum acceptable concentration EQS as an absolute upper concentration limit is likely to result in a substantial waste of resources due to chasing down the wrong management priorities.

Current EU standards lack a spatial dimension. Water authorities are allowed to define a transitional area of exceedance (CEC 2006), but guidelines for spatial and temporal specification of this EQS exceedance are lacking. Adequate regulation of mixing zones requires more research on the relationship between the spatial scale of water pollution and the impact on aquatic ecosystems and human health. Dutch soil regulations may serve as an example. Here, it is determined that the applicable soil standard should be exceeded in at least $25 \mathrm{~m}^{3}$ of soil before remediation is considered (Staatscourant 2000). In the meantime, a provision could be adopted that mixing zones should not impair human health or the ecological integrity of the water system.

\section{Case IV: Quantification of uncertainty to optimize the allocation of monetary resources}

Ragas et al. (2005) performed a case study in which the costs of reducing true uncertainty in ecological sediment standards were compared to the potential benefits. The case study dealt with DDT-contaminated sediments. In the Netherlands, polluted sediments are classified based on EQSs. Moderately and heavily polluted sediment (classes 3 and 4) must be disposed of at a cost of approximately $€ 65.00 / \mathrm{m}^{3}$. For clean and slightly polluted sediment (classes 0-2), the costs are approximately $€ 7.70 / \mathrm{m}^{3}$; a difference of $€ 57.30$ / $\mathrm{m}^{3}$. The water board Rivierenland in the Netherlands processes approximately $63000 \mathrm{~m}^{3}$ of class 3 and 4 sediments yearly, which are mainly polluted with DDT from intensive fruit culture. It is obvious that a considerable amount of money could be saved if more sediment was classified in class 2 instead of class 3 or 4 .

An EQS is used to distinguish between classes 2 and 3. This EQS was derived as the geometric mean of the hazardous concentration for $50 \%$ and $5 \%$ of the species $\left(\mathrm{HC}_{50}\right.$ and $\mathrm{HC}_{5}$ ). These values were calculated using a SSD based on 3 chronic NOEC values; one each for algae, daphnia, and fish (Aldenberg and Jaworska 2000). Species sensitivity distributions provide a relatively conservative estimate of the $\mathrm{HC}_{5}$ when data input is low because the estimated interspecies variability is slightly biased due to the low number of input data. This means that the $\mathrm{HC}_{5}$ tends to increase when the number of available NOECs increases. This raises the question of what would happen to the EQS of DDT if a 4 th NOEC would be added to the current dataset. Ragas et al. (2005) explored this issue by generating new hypothetical NOEC values based on 2 different methods: 1) parametric 
bootstrapping of the existing data set, and 2) the analysis of species-specific patterns in a database with NOEC values. The first option showed an $80 \%$ probability to obtain a less stringent $\mathrm{HC}_{5}$ value, and the second option a $92 \%$ probability. The expected benefits due to reduced disposal costs in the second option were estimated at $€ 1.9$ million per year. The average costs of an ecotoxicity test are approximately $€ 40000$. A more elaborate study on the impact of new ecotoxicity data on sediment standards was performed by Henning-De Jong et al. (2008).

The example shows that investment in an extra toxicity test can result in considerable benefits. However, this only works if the amount of uncertainty in the assessment is implicitly or explicitly regulated; for example, by using the 90th percentile of a probability distribution as a standard. More data will mean smaller confidence intervals and thus less stringent standards. It is interesting to note that this does not always apply to the derivation of EQSs under the WFD. Here, safety factors are generally used to derive EQSs. If more than 3 and less than 10 NOECs are available, a safety factor of 10 is applied to the lowest NOEC. In this specific case, an extra NOEC can never result in a less stringent EQS, only in a more stringent. This is an example of inconsistent regulation of uncertainty and not in line with the general regulation principle that safety margins should decrease when knowledge increases.

\section{UNCERTAINTY AND RISK MANAGEMENT}

The case studies presented illustrate how different types of uncertainty and variability can be described or quantified. But how can the risk manager use this information to improve risk management? This question is addressed in this section.

\section{Problem definition uncertainty}

The case studies presented here did not include the analysis of problem definition uncertainty. Actually, very few studies on problem definition uncertainty are available in the field of environmental risk assessment. Thomsen et al. (2006) outline a framework for identifying and describing problem definition uncertainty, but this method has yet been little applied in practice. Furthermore, it is based on the assumption that an environmental problem can be exactly defined, which is a questionable assumption as will be pointed out below.

Problem definition uncertainty can be avoided by a careful and comprehensive analysis of the management problem at hand and subsequent translation into one or more scientific questions. It is rarely possible to fully cover a risk assessment problem in a single scientific question or study; gaps in knowledge will most likely remain. The risk assessor and risk manager should be aware of those gaps in order to avoid misunderstanding and the false impression that the risk assessment problem can be solved once scientists have done their job. Most importantly, this implies that the problem owner (i.e., the risk manager) and the scientist (i.e., the risk assessor) should communicate clearly about the scope of the problem definition and the scientific conclusions.

An example where problem definition uncertainty was appropriately dealt with can be obtained from the EU WFD. Before introduction of the WFD, water quality management in most European states focused primarily on chemical water quality, such as the maintenance of EQSs for single substances. The WFD introduced the concept of a "good ecological status" which includes the monitoring of biological parameters. This reflects awareness that the protection of aquatic ecosystems (i.e., the management problem) is more comprehensive than the maintenance of a good chemical status (i.e., the risk assessment problem).

An interesting perspective on problem definition uncertainty within the context of the WFD has been put forward by Newig et al. (2005). They use the term normative uncertainty, that is, normative considerations of goals and tastes. An actor may be in doubt (or a group of actors may be undecided) as to what goal to pursue and what actions to take in order to achieve these goals. Newig et al. (2005) emphasized the fact that the definition of a management problem depends on the perspectives and interests of the actors involved; there does not exist one problem definition but a range of possible problem definitions. This puts problem definition uncertainty as defined in the present paper (i.e., the management problem is not fully covered by the scientific analysis) in another perspective. If there is ambiguity about the definition of the management problem, this will also impact the scientific analysis. Newig et al. (2005) proposed a participatory approach as a way to dealing with normative uncertainty. Actually, the WFD is one of the first European regulations that explicitly demand a high degree of involvement of nonstate actors in the implementation. Possible techniques to design a participatory process where different perspectives can be expressed and combined with factual analyses are the use of group model building and of participatory scenario development (Van der Heijden 1996; Pahl-Wostl 2005). These techniques structure the process of goal determination and make the steps required to achieve these goals more transparent. We would like to stress the importance of involving the actual risk assessors (i.e., the scientists) in the participatory process. Through their involvement they will gain a better understanding of the problem at hand which facilitates the scientific analysis. Furthermore, they can indicate gaps of knowledge during an early phase of the process, which may prevent unrealistic expectations of nonexpert actors involved.

\section{True uncertainty}

The case studies illustrated various ways to describe and quantify true uncertainty in risk assessment, such as Monte Carlo simulation, model comparisons, and expert judgment. Once true uncertainty has been described or quantified, the question rises of how it can be used in risk management. Several approaches are possible for dealing with true uncertainty. Which approach to take depends on how accurately uncertainty can be described or quantified. Three approaches are discussed below. They are referred to as the approach of equal probability, the tiered approach, and the participatory approach.

Approach of equal probability-The approach of equal probability is based on the supposition that true uncertainty can be quantified objectively and should be treated in a consistent manner, that is, each standard or risk estimate should reflect an equal level of uncertainty. An example is the convention that the median value of the predicted $\mathrm{HC}_{5}$ from a SSD is taken as the ecological standard (Lepper 2005). The use of the median implies that each standard reflects the same amount of true uncertainty. The first case study showed that this is currently not always the case-the level of uncertainty in the virtually safe doses of acrylamide, chlordane, and DDT varied considerably. This also applies to the derivation of 
ecological standards according to the WFD if 3 to 9 NOECs are available. The level of true uncertainty in assessing ecosystem sensitivity decreases with an increasing number of NOECs, but the PNEC derived with safety factors is likely to decrease. Given the fact that less than 10 NOEC values are available for most chemicals, it is obvious that most ecological standards derived according to the WFD guidelines reflect a varying level of true uncertainty.

The approach of equal probability requires that true uncertainty be expressed in probabilistic terms, but has the advantage that true uncertainty can be regulated in a consistent manner. Moreover, it creates the opportunity to explicitly define a level of conservativeness in risk assessment that rewards the gathering of new information. This was demonstrated in the case study on polluted sediments (i.e., a trend was shown that gathering of extra toxicity data generally results in less stringent standards). However, in this particular case, the reduction in uncertainty was the result of a biased estimate of the variance at low sample sizes (see Case IV) and not of explicit regulation of true uncertainty. A more explicit regulation of true uncertainty can have considerable advantages from a risk management perspective, especially within the context of the new European Union chemical regulations (i.e., Registration, Evaluation, Authorization and restriction of Chemical substances [REACH]). For example, if standards would be defined as the 90th percentile of a probability distribution, this would stimulate the gathering of extra toxicity data because this extra information is likely to result in less stringent standards without jeopardizing the level of confidence in the protection level.

Tiered approach - In a tiered approach, relatively simple risk assessment methods are applied when few data are available and more complex and realistic methods when data availability increases (Cowan et al. 1995; Van Leeuwen and Hermens 1995; USEPA 1998). It probably is the most widely used approach to deal with true uncertainty in risk assessment. Elements of this approach can be found in the guidelines for derivation of WFD standards (Lepper 2005) and the European Union TGD guidelines for the evaluation of new and existing substances (EC 2003). An example is the application of safety factors to derive ecological standards if less than 10 NOECs are available and the application of SSDs when more NOECs are available.

A tiered approach is an implicit way to regulate true uncertainty. Instead of regulating the level of true uncertainty, as in the approach of equal probability, it is prescribed which risk assessment methods should be used, depending on data availability. The exact level of true uncertainty in the assessment remains unknown, but there is a general instinctive consensus that the estimated risk becomes more realistic when the assessment methods become more sophisticated. It is often argued that the outcome of a simple risk assessment is "more conservative" than the outcome of a sophisticated assessment (Van Leeuwen and Hermens 1995). This is true if "more conservative" is interpreted as "further from the real value," but it should be stressed that the actual probability level of the outcome of a simple risk assessment is not necessarily lower than that of a more sophisticated method. Both estimates may have a similar probability level given the available information (e.g., the 90th percentile of a probability distribution), but the outcome of the sophisticated method will be closer to reality because the variance in the probability distribution (reflecting the uncertainty in the assessment) is smaller.
Participatory approach-In some risk assessments, the level of true uncertainty may be difficult to describe or quantify, such as the huge uncertainty in the low-dose extrapolation of genotoxic carcinogens in the first case study. The uncertainty estimate strongly depends on subjective choices such as the dose-response models included in the study. This type of uncertainty may result in intense controversies between the stakeholders, especially when the stakes involved are high. An example would be the assessment of health risks due to the consumption of fish polluted with dioxins in a region where cancer incidence is relatively high. Many scientific controversies exist in the risk assessment of dioxins (i.e., about whether it causes cancer and at what exposure levels) (Kaiser 2000). Fish consumers living in the affected region will most likely assess the risk in a different way than fishermen or those responsible for the $\mathrm{PCB}$ contamination. In this type of controversy, a tiered approach or an approach of equal probabilities is often unfeasible and nonproductive. The stakeholders involved will tend to emphasize the (unknown) uncertainties that threaten their own interests. Lack of consensus on how to deal with these uncertainties can frustrate the decision-making process and hamper the implementation of policy measures.

Conflicts between stakeholders on how to interpret and deal with (unknown) true uncertainty are essentially conflicts of interests. This has led scientists to plead for the involvement of stakeholders in the problem analysis or, in other words, participatory problem analysis (Funtowicz and Ravetz 1994; Hellström 1996). The aim of involving the stakeholders is not to reduce or quantify true uncertainty, but to identify all potential sources of true uncertainty and to deal with it in a way that is acceptable to all stakeholders. Numerous studies have shown that "lay local knowledge" can add valuable insights into the analysis of the problem (Wynne 1992; Pellizzoni 2003; Yearley et al. 2003).

Since a participatory approach was also proposed to deal with problem definition uncertainty, such an approach can serve multiple aims:

1. The formulation of a problem definition that is acceptable to all stakeholders;

2. The identification of sources of true uncertainty in the risk assessment process;

3. Identifying ways to deal with true uncertainty and solve the management problem in a way that is acceptable to all stakeholders.

\section{Variability}

Ways of dealing with variability in risk management depend on the type of variability. Most of them have already been addressed in the case studies presented above. They can be summarized as follows:

- Interindividual variability in human risk assessment relates to the population fraction at risk of exceeding a certain exposure or effect level. What is considered acceptable should be determined by society in a process of political decision making. Information about interindividual variability enables the risk manager to take specific actions to reduce the risk for subpopulations with a high risk level.

- Interspecies variability in ecological risk assessment relates to the fraction of species at risk of exceeding a certain exposure or effect level. If the aim is to "protect 
ecosystem integrity," it requires scientific analysis to determine at what "fraction of species affected" ecosystem integrity is impaired. In practice, ecological standards are often based on the premise that ecosystem integrity will not be impaired if the NOEC is exceeded for no more than $5 \%$ of the species (i.e., the $\mathrm{HC}_{5}$ ). This premise has been heavily criticized on theoretical grounds (Forbes and Forbes 1993), but several mesocosm and field studies have shown no or negligible adverse effects at the level of the $\mathrm{HC}_{5}$ (Van den Brink et al. 2002, 2006; Schroer et al. 2004; Maltby et al. 2005).

- Spatial and temporal variability relate to questions such as "How do spatial and temporal concentration variations influence risks?" This applies to human as well as ecological risk assessment. The current scientific basis for dealing with spatial and temporal concentration variations is weak, especially in effect assessment. In order to improve this, more research is necessary on how spatial and temporal concentration variations impact human health and ecosystem integrity. Information on spatial and temporal variability can support the identification of hot spots and risk peaks in time, respectively.

- Some types of variability in risk assessment can result in true uncertainty. For example, interspecies variability data are often used to indicate the uncertainty in the extrapolation of laboratory animal data to humans. Another example is the variability between chemical substances which is often used as a measure of uncertainty in the prediction of toxicity for untested substances.

\section{CONCLUSIONS AND RECOMMENDATIONS}

This paper presented a typology of uncertainty, distinguishing problem definition uncertainty, true uncertainty, and variability. Different ways to describe and quantify these types of uncertainty were discussed and most of them were illustrated in the case studies. It was demonstrated that addressing the different types of uncertainty can improve risk management, such as:

- Problem definition uncertainty can result in risk assessments that do not fully address the management problem at hand. This can be prevented by a participatory approach (i.e., involving stakeholders and risk assessors in the definition of the management problem and the subsequent translation into scientific questions).

- True uncertainty can be described or quantified by means of various techniques, such as Monte Carlo simulation, model comparison, and expert elicitation. True uncertainty that can be expressed probabilistically provides the opportunity to harmonize the level of true uncertainty in risk assessments and to apply uncertainty as a management tool to stimulate the gathering of new information. However, reliable expression of true uncertainty in probabilistic terms is not always feasible. Alternative ways to deal with true uncertainty are a tiered risk assessment approach and a participatory approach.

- Variability analysis can provide insight in the variation of risks between individuals, species, locations, moments in time, and substances. This information enables the risk manager to take targeted measures to reduce the risk where it is highest.

The European Union WFD provides various opportunities to address uncertainty in risk-based management of river basins. It is one of the first European regulations that explicitly demand a high degree of nongovernmental actors in river basin management, which may be particularly helpful when dealing with problem definition uncertainty and forms of true uncertainty that are difficult to quantify. The European Union WFD is less advanced when it comes to regulation of probabilistic uncertainty and various types of variability. The guidelines for derivation of EQSs (Lepper 2005) stick to a tiered approach risk assessment. We have shown in our case studies that this can result in standards with a varying level of true uncertainty. Furthermore, guidelines to quantify and deal with different forms variability are few and the scientific basis for them is weak, especially when it comes to interindividual, spatial, and temporal variability.

Acknowledgment-This research was financially supported by the European Union (European Commission, FP6 project NoMiracle, Contract 003956).

\section{REFERENCES}

Aldenberg T, Jaworska JS. 2000. Uncertainty of the hazardous concentration and fraction affected for normal species sensitivity distributions. Ecotoxicol Environ Saf 46:1-18.

Bogen KT, Spear RC. 1987. Integrating uncertainty and interindividual variability in environmental risk assessment. Risk Anal 7:427-436.

[CEC] Commission of the European Communities. 2006. Directive of the European parliament and of the council on environmental quality standards in the field of water policy and amending Directive 2000/60/EC. Brussels (BE): CEC. $\operatorname{COM}(2006) 397$ final, 2006/0129 (COD).

Cowan CE, Versteeg DJ, Larson RJ, Kloeppersams PJ. 1995. Integrated approach for environmental assessment of new and existing substances. Reg Toxicol Pharmacol 21:3-31.

Dorne JLCM, Skinner L, Frampton G, Spurgeon D, Ragas AMJ. 2007. Human and environmental risk assessment of pharmaceuticals: differences, similarities, lessons from toxicology. Anal Bioanal Chem 387:1259-1268.

Dorne JLCM, Walton K, Renwick AG. 2005. Human variability in xenobiotic metabolism and pathway-related uncertainty factors for chemical risk assessment: a review. Food Chem Toxicol 43:203-216.

[EC] European Commission. 2003. Technical guidance document on risk assessment. Luxemburg (LU): Office for Official Publications of the European Communities.

Evans JS, Gray GM, Sielken RL, Smith AE, Valdez FC, Graham JD. 1994. Use of probabilistic expert judgment in uncertainty analysis of carcinogenic potency. Reg Toxicol Pharmacol 20:15-36.

Forbes TL, Forbes VE. 1993. A critique of the use of distribution-based extrapolation models in ecotoxicology. Funct Ecol 7:249-254.

Frey HC. 1992. Quantitative analysis of uncertainty and variability in environmental policy making. Pittsburgh (PA): Research Associate Center for Energy and Environmental Studies.

Funtowicz SO, Ravetz JR. 1990. Uncertainty and quality in science for policy. Dordrecht (NL): Kluwer Academic.

Funtowicz SO, Ravetz JR. 1994. The worth of a songbird: ecological economics as a post-normal science. Ecol Econ 10:197-208.

Hammersley JM, Handscomb DC. 1964. Monte Carlo methods. New York (NY): John Wiley and Sons.

Hellström T. 1996. The science-policy dialogue in transformation: Modeluncertainty and environmental policy. Science and Public Policy 23:91-97.

Henning-De Jong I, Ragas AMJ, Hendriks HWM. 2008. Report on the impact of an additional ecotoxicity test when deriving environmental quality standards. Nijmegen (NL): Radboud University. NoMiracle Deliverable 4.1.8.

Hollander A. 2008. Spatial variation in multimedia mass balance models: The relative influence of environmental characteristics on the spatial variation in predicted environmental concentrations of chemicals [PhD Thesis]. Nijmegen (NL): Radboud Univ.

Huijbregts MAJ, Geelen LMG, Hertwich EG, McKone TE, Van de Meent D. 2005. A comparison between the multimedia fate and exposure models CalTOX and uniform system for evaluation of substances adapted for life-cycle assessment based on the population intake fraction of toxic pollutants. Environ Toxicol Chem 24:486-493. 
Huijbregts MAJ, Gilijamse W, Ragas AMJ, Reijnders L. 2003. Evaluating uncertainty in environmental life-cycle assessment: A case study comparing two insulation options for a Dutch one-family dwelling. Environ Sci Technol 37:2600-2608.

Kaiser J. 2000. Dioxin draft sparks controversy. Science 288:1313-1314.

Kelly EJ, Campbell K. 2000. Separating variability and uncertainty in environmental risk assessment-Making choices. Human Ecol Risk Assess 6:1-13.

Lepper P. 2005. Manual on the methodological framework to derive environmental quality standards for priority substances in accordance with Article 16 of the Water Framework Directive (2000/60/EC). Schmallenberg (DE): Fraunhofer-Institute.

Leuven RSEW, Poudevigne I. 2002. Riverine landscape dynamics and ecological risk assessment. Freshwater Biol 47:845-865.

Maltby L, Blake N, Brock TCM, Van den Brink PJ. 2005. Insecticide species sensitivity distributions: Importance of test species selection and relevance to aquatic ecosystems. Environ Toxicol Chem 24:379-388.

McKone TE, Ryan PB. 1989. Human exposures to chemicals through food chains: An uncertainty analysis. Environ Sci Technol 23:1154-1163.

Morgan MG, Henrion M. 1990. Uncertainty: A guide to dealing with uncertainty in quantitative risk and policy analysis. Cambridge (UK): Cambridge University Press.

Newig J, Pahl-Wostl C, Sigel K. 2005. The role of public participation in managing uncertainty in the implementation of the Water Framework Directive. Eur Env 15:333-343.

[NRC] National Research Council. 1975. Reactor safety: An assessment of accident risks in the U.S. commercial nuclear power plants. Washington DC: Nuclear Regulatory Commission. NUREG-75-014 (WASH-1400).

[NRC] National Research Council. 1994. Science and judgment in risk assessment. Washington DC: National Academy Press.

Pahl-Wostl C. 2005. Information, public empowerment and the management of urban watersheds. Environ Model Assess 20:457-467.

Pellizzoni L. 2003. Uncertainty and participatory democracy. Environmental Values $12: 195-224$

Ragas AMJ. 1999. The consequences of applying different mixing models, input data, and EQOs when deriving emission limits for discharge permits. European Water Management 2:62-71.

Ragas AMJ. 2000. Uncertainty in environmental quality standards [PhD thesis] Nijmegen (NL): Radboud Univ.

Ragas AMJ, Boomsma D, Huijbregts MAJ. 2005. Quantifying the benefits of an extra ecotoxicity test to derive PNECs. In: Society of Environmental Toxicology and Chemistry (SETAC) 15th Annual Meeting; 22-26 May 2005, SETAC Europe, Brussels. Lille (FR): SETAC.

Ragas AMJ, Brouwer FPE, Büchner FL, Hendriks HWM, Huijbregts MAJ. 2008. Separation of uncertainty and interindividual variability in human exposure modeling. Journal of Exposure Science and Environmental Epidemiology (in press).

Ragas AMJ, Etienne RS, Willemsen FH, Van de Meent D. 1999. Assessing model uncertainty for environmental decision making: A case study of the coherence of independently derived environmental quality objectives for air and water. Environ Toxicol Chem 18:1856-1867.

Ragas AMJ, Huijbregts MAJ. 1999. Evaluating the coherence between environmental quality objectives and the acceptable or tolerable daily intake. Regul Toxicol Pharmacol 27:251-264.

Renn O. 1998. The role of risk perception for risk management. Reliability Engineering and System Safety 59:49-62.
Schroer AFW, Belgers JDM, Brock TCM, Matser AM, Maund SJ, Van den Brink PJ. 2004. Comparison of laboratory single species and field population-level effects of the pyrethroid insecticide $\lambda$-cyhalothrin on freshwater vertebrates. Arch Environ Contam Toxicol 26:324-335.

Slob W. 1994. Uncertainty analysis in multiplicative models. Risk Analysis 14:571576.

Slovic P. 2001. The risk game. J Hazard Mater 86:17-24.

Staatscourant 2000. Circulaire Streef-en interventiewaarden bodemsanering. Den Haag (NL): SDU. Staatscourant 2000:39 (in Dutch).

Thiessen KM, Hoffman FO, Rantavaara A, Hossain S. 1997. Environmental models undergo international test: The science and art of exposure assessment modeling were tested using real-world data from the Chernobyl accident. Environ Sci Technol 31:358-363.

Thomsen M, Sorensen PB, Fauser P, Faber J, Van der Pol J, Peirano F, Vernai AM, Strebel K, Schlink U, Espinosa Porragas G, Giralt i Prat F, Rallo R, Pistocchi A. 2006. Pre-selection of scenario for cumulative risk assessment-Documentation of the first version of selection procedure including initial uncertainty evaluation. Silkeborg (DK): National Environmental Research Institute. NoMiracle Deliverable 1.2.4

[USEPA] U.S. Environmental Protection Agency. 1998. Guidelines for ecological risk assessment. EPA/630/R-95/002F. Washington DC: Risk Assessment Forum. Federal Register 63:26846-26924.

[USPCCRARM] U.S. Presidential/Congressional Commission on Risk Assessment and Risk Management. 1997. Framework for environmental health risk management. Washington DC: USPCCRARM.

Van Asselt MBA, Rotmans J, Rothman DS, eds. 2005. Scenario innovation: Experiences from a European experimental garden. London (UK): Taylor and Francis.

Van den Brink PJ, Blake N, Brock TCM, Maltby L. 2006. Predictive value of species sensitivity distributions for effects of herbicides in freshwater ecosystems. Human Ecol Risk Assess 12:645-764.

Van den Brink PJ, Brock TCM, Posthuma L. 2002. The value of the species sensitivity distribution concept for predicting field effects: (Non-)confirmation of the concept using semifield experiments. In: Posthuma L, Suter GW II, Traas TP, editors. Species sensitivity distributions in ecotoxicology. Boca Raton (FL): Lewis. p 155-193.

Van der Heijden K. 1996. Scenarios: The art of strategic conversation. Chichester (UK): Wiley.

Van der Sluijs JP, Craye M, Funtowicz S, Kloprogge P, Ravetz J, Risbey J. 2005 Combining quantitative and qualitative measures of uncertainty in modelbased environmental assessment: The NUSAP System. Risk Analysis 25:481492.

Van Leeuwen CJ, Hermens JLM. 1995. Risk assessment of chemicals: An introduction. Dordrecht (NL): Kluwer Academic. 374 p.

Vercelli A. 1995. From soft uncertainty to hard environmental uncertainty. Economie Appliquée 48:251-270.

Webster M, Forest C, Reilly J, Babiker M, Kicklighter D, Mayers M, Prinn R, Sarofim M, Sokolov A, Stone P, Wang C. 2003. Uncertainty analysis of climate change and policy response. Climatic Change 61:295-320.

Wynne B. 1992. Misunderstood misunderstanding: Social identities and public uptake of science. Public Understanding of Science 1:281-304

Yearley S, Cinderby S, Forrester J, Bailey P, Rosen P. 2003. Participatory modelling and the local governance of the politics of UK air pollution: A three-city case study. Environmental Values 12:247-262 